\title{
PRECISE LARGE DEVIATIONS FOR ORNSTEIN-UHLENBECK PROCESSES*
}

\author{
XIANGFENG YANG
}

\begin{abstract}
For a family of Ornstein-Uhlenbeck processes having an infinitesimal size of noise, we prove precise asymptotics for large deviations of an integral form over the continuous path space. The main ingredients in the proof are an exponential change of measures and subtle Taylor expansions with suitable estimates. An application of these precise integral large deviations to partial differential equations is included.
\end{abstract}

\section{Introduction}

In this paper, we consider Ornstein-Uhlenbeck (OU) processes $\left\{U^{\lambda}\right\}_{0 \leq t \leq 1}$ on some probability space $(\Omega, \mathscr{F}, \mathbf{P})$ with a small size of magnitude $O\left(\lambda^{-1}\right)$. Namely, for each fixed $\lambda$, the process $U^{\lambda}$ is determined as the unique solution to the stochastic differential equation

$$
d U_{t}^{\lambda}=-U_{t}^{\lambda} d t+\lambda^{-1} d W_{t}, \quad U_{0}^{\lambda}=0 .
$$

The main subject of this paper is to study the precise asymptotics as $\lambda \rightarrow \infty$ for the expectation $\mathbf{E} \exp \left\{\lambda^{2} F\left(U^{\lambda}\right)\right\}$ under suitable assumptions on the function $F$ over the continuous function space $C[0,1]$. This is one form of large deviations.

Large deviations in limit theorems can be formulated as follows. Let $\mathbf{X}$ be a metric space with metric $\rho$, and $\mu^{\varepsilon}$ be a family of probability measures on $\mathbf{X}$ depending on a parameter $\varepsilon>0$. Suppose there is a point $x_{*} \in \mathbf{X}$ such that for any $\delta>0$ and small $\varepsilon, \mu^{\varepsilon}\left\{y: \rho\left(x_{*}, y\right)<\delta\right\}$ have overwhelming probabilities: $\lim _{\varepsilon \rightarrow 0} \mu^{\varepsilon}\left\{y: \rho\left(x_{*}, y\right)<\delta\right\}=1$. Problems on large deviations are concerned with the limiting behavior as $\varepsilon \rightarrow 0$ of the small probabilities $\mu^{\varepsilon}(A)$ for measurable sets $A \subseteq \mathbf{X}$ that are situated at a positive distance from point $x_{*}$ (cf. [1]). Problems concerning asymptotics as $\varepsilon \rightarrow 0$ of integrals in the form $\int_{\mathbf{X}} f_{\varepsilon}(x) \mu^{\varepsilon}(d x)$ also

* Research supported by the National Science Foundation of China (NSFC) 11226209.

2010 Mathematics Subject Classification. Primary 60F10; Secondary 60J65, 35C10.

Key words and phrases. Large deviation, Ornstein-Uhlenbeck process, exponential change of measures.

Received December 11, 2012; revised March 1, 2013. 
belong to large deviations if the main part of such integrals for small $\varepsilon$ is due to the values of $x$ far away from point $x_{*}$ (cf. [8]).

The study on large deviations of integral forms for stochastic processes $\xi_{t}^{\varepsilon}$ defined on probability spaces $\left(\Omega, \mathscr{F}, \mathbf{P}^{\varepsilon}\right)$ is generally obtained in the sense of logarithmic equivalence: $\ln \mathbf{E}^{\varepsilon} f_{\varepsilon}\left(\xi^{\varepsilon}\right) \sim g\left(\varepsilon, f_{\varepsilon}\right)$, where we use $\mathbf{E}^{\varepsilon}$ to denote the mathematical expectation with respect to measure $\mathbf{P}^{\varepsilon}$ (see [2], [7] and [8]). These results are generally called rough large deviations. It is natural to expect more precise large deviations under more assumptions on the processes and the functional. Indeed, for the family of stochastic processes $\left\{\sqrt{\varepsilon} W_{t}\right\}_{0 \leq t \leq 1}$ with $W$ denoting the standard Wiener process, Schilder showed in [6] the following precise large deviations

$$
\mathbf{E} \exp \left\{\varepsilon^{-1} F(\sqrt{\varepsilon} W)\right\}=\exp \left\{\varepsilon^{-1}\left[F\left(\phi_{0}\right)-S\left(\phi_{0}\right)\right]\right\}\left[\sum_{0 \leq i \leq s / 2} K_{i} \cdot \varepsilon^{i}+o\left(\varepsilon^{s / 2}\right)\right]
$$

for some positive integer $s$ depending on the smoothness of $F$, where the normalized action functional $S(\phi)=\frac{1}{2} \int_{0}^{1} \phi^{\prime}(t)^{2} d t$ for absolutely continuous $\phi$ and $S(\phi)=\infty$ for other $\phi$, and $\phi_{0}$ is the unique maximizer of $F-S$ over the continuous path space. Then Ellis and Rosen in [3] proved similar precise large deviations in the setting of Hilbert spaces for some Gaussian probability measures (see also [5]). In this paper, we aim to prove the same type of precise large deviations for $\mathbf{E} \exp \left\{\lambda^{2} F\left(U^{\lambda}\right)\right\}$.

The main result of this paper is formulated in Section 2. An exponential change of measures for Markov processes is included in Section 3.1, which serves as the main tool for the proof. Section 3.3 contains an application of these precise large deviations to partial differential equations.

\section{The main result}

We use $C_{0}[0,1]$ to denote the space of all continuous functions on $[0,1]$ vanishing at zero, and $C_{0}^{1}[0,1]$ the space of continuously differentiable functions vanishing at zero. On these spaces, the metric $\rho$ induced by the uniform norm $\|\cdot\|$ will be used:

$$
\rho(\phi, \varphi)=\|\phi-\varphi\|=\max _{0 \leq t \leq 1}|\phi(t)-\varphi(t)| .
$$

OU process $\left\{U^{\lambda}\right\}_{0 \leq t \leq 1}$ defined by $(1.1)$ is a Markov process with the generating operator

$$
A^{U^{\lambda}} f(x)=-x f^{\prime}(x)+\frac{1}{2} \lambda^{-2} f^{\prime \prime}(x)
$$

We introduce the cumulant $G^{U^{\lambda}}$ of $U^{\lambda}$ as follows

$$
G^{U^{\lambda}}(x ; z)=-z x+\frac{1}{2} \lambda^{-2} z^{2}=\lambda^{2} G_{0}\left(x ; \lambda^{-2} z\right)
$$


with $G_{0}(x ; z)=-z x+\frac{1}{2} z^{2}$. The Legendre transformation $H_{0}(x ; u)$ of $G_{0}(x ; z)$ in its second argument is defined by:

$$
H_{0}(x ; u)=\sup _{z \in \mathbf{R}}\left[z \cdot u-G_{0}(x ; z)\right]=\frac{1}{2}(x+u)^{2} .
$$

Now we define the normalized action functional $S$ over the space $C_{0}[0,1]$ as

$$
S(\phi)=\int_{0}^{1} H_{0}\left(\phi(t) ; \phi^{\prime}(t)\right) d t
$$

for absolutely continuous function $\phi$, and $S(\phi)=\infty$ otherwise. This functional is lower semi-continuous (cf. Section 3.1 in [8]).

THEOREM 2.1. Let $F$ be a measurable functional on $C_{0}[0,1]$ which is bounded above. Suppose that the maximum of the functional $F-S$ over the space $C_{0}[0,1]$ is attained at a unique function $\phi_{0} \in C_{0}^{1}[0,1]$, and that $F$ is s times Fréchet differentiable at $\phi_{0}$ in the uniform topology with $F^{\prime \prime}\left(\phi_{0}\right)(\phi, \phi) \leq 0$ for any $\phi \in C_{0}[0,1]$. Then as $\lambda \rightarrow \infty$,

$$
\mathbf{E} \exp \left\{\lambda^{2} F\left(U^{\lambda}\right)\right\}=\exp \left\{\lambda^{2}\left[F\left(\phi_{0}\right)-S\left(\phi_{0}\right)\right]\right\}\left[\sum_{0 \leq i \leq s-2} K_{i} \lambda^{-i}+o\left(\lambda^{-(s-2)}\right)\right]
$$

where the coefficients $K_{i}$ are determined by $F$ and its derivatives at $\phi_{0}$; in particular $K_{0}=\mathbf{E} \exp \left\{\frac{1}{2} F^{\prime \prime}\left(\phi_{0}\right)\left(U^{1}, U^{1}\right)\right\}$.

\section{The proof and an application}

\subsection{Exponential change of measures}

Following [8], we here define a new probability measure which is an exponential change of the original probability measure $\mathbf{P}$. To this end, we introduce functions

$$
z^{\lambda}(t)=\lambda^{2} z_{0}(t), \quad \text { where } z_{0}(t)=\frac{\partial H_{0}}{\partial u}\left(\phi_{0}(t) ; \phi_{0}^{\prime}(t)\right)=\phi_{0}(t)+\phi_{0}^{\prime}(t) .
$$

The new family of probability measures is defined as

$$
\mathbf{P}^{z^{\lambda}}(A):=\int_{A} \exp \left\{\int_{0}^{1} z^{\lambda}(t) d U_{t}^{\lambda}-\lambda^{2} \int_{0}^{1} G_{0}\left(U_{t}^{\lambda} ; z_{0}(t)\right) d t\right\} d \mathbf{P} .
$$

For each $\lambda>0$, under the new probability measure $\mathbf{P}^{z^{\lambda}}$, the process $U^{\lambda}$ is again a Markov process with the generating operator (cf. Section 2.2 in [8])

$$
A_{t}^{z^{\lambda}} f(x)=\frac{\partial G_{0}}{\partial z}\left(x ; z_{0}(t)\right) f^{\prime}(x)+\frac{1}{2} \lambda^{-2} f^{\prime \prime}(x)
$$


Then it turns out that the normalized process $\eta_{t}^{\lambda}:=\lambda\left(U_{t}^{\lambda}-\phi_{0}(t)\right)$ as a Markov process has the generating operator

$$
A^{\eta^{\lambda}} f(x)=-x f^{\prime}(x)+\frac{1}{2} f^{\prime \prime}(x)
$$

from which it follows that $\eta^{\lambda}$ under $\mathbf{P}^{z^{\lambda}}$ and $U^{1}$ under $\mathbf{P}$ have the same distribution on the space $C_{0}[0,1]$.

The proof of Theorem 2.1 will rest on the next lemma. For every $s>0$, put

$$
\Phi(s)=\left\{\phi: \phi \in C_{0}[0,1] \text { and } S(\phi) \leq s\right\} .
$$

The set $\Phi(s)$ is compact in $C_{0}[0,1]$ (cf. Section 3.1 in [8]).

Lemma 3.1. (i) For any $\delta>0, \gamma>0$ and $s_{0}>0$, there exists $\lambda_{0}>0$ such that

$$
\mathbf{P}\left\{\rho\left(U^{\lambda}, \phi\right)<\delta\right\} \geq \exp \left\{-\lambda^{2}[S(\phi)+\gamma]\right\}
$$

for any $\lambda>\lambda_{0}$ and any $\phi \in \Phi\left(s_{0}\right)$;

(ii) For any $\delta>0, \gamma>0$ and $s_{0}>0$, there exists $\lambda_{0}>0$ such that

$$
\mathbf{P}\left\{\rho\left(U^{\lambda}, \Phi(s)\right) \geq \delta\right\} \leq \exp \left\{-\lambda^{2}(s-\gamma)\right\}
$$

for any $\lambda>\lambda_{0}$ and any $s \leq s_{0}$.

Remark 3.2. Lemma 3.1 shows that the family of $U^{\lambda}$ satisfies a large deviation principle. There are several equivalent definitions for a large deviation principle (see Section 3.3 in [4] and the Introduction in [8]), and here we adopt the definition in [4]. Formally speaking, the large deviation principle in Lemma 3.1 states that as $\lambda \rightarrow \infty$, the process $U^{\lambda}$ will stick around the path $\phi_{*}$ such that $S\left(\phi_{*}\right)=0$. Away from the most probable path $\phi_{*}$, the probability tends to zero exponentially fast.

Remark 3.3. The proof of this lemma is a direct application of Theorem 5.6.3 in [2]. More precisely, large deviation principles are proved in [2] for more general families of processes $X^{\varepsilon}$ determined by

$$
d X_{t}^{\varepsilon}=b\left(X_{t}^{\varepsilon}\right) d t+\sqrt{\varepsilon} d W_{t}, \quad 0 \leq t \leq 1, X_{0}^{\varepsilon}=0
$$

with a uniformly Lipschitz continuous function $b(\cdot): \mathbf{R} \rightarrow \mathbf{R}$.

\subsection{Taylor's expansions}

From Lemma 3.1 and the assumption that $F$ is bounded above, it follows that for any $\varepsilon>0$, there exists some $\gamma>0$ such that as $\lambda \rightarrow \infty$ (cf. Lemma 0.1 in [8]),

$$
\begin{aligned}
& \mathbf{E} \exp \left\{\lambda^{2} F\left(U^{\lambda}\right)\right\} \\
& \quad=\mathbf{E}\left[1_{\left\{\left\|U^{\lambda}-\phi_{0}\right\|<\varepsilon\right\}} \exp \left\{\lambda^{2} F\left(U^{\lambda}\right)\right\}\right]+o\left(\exp \left\{\lambda^{2}\left[F\left(\phi_{0}\right)-S\left(\phi_{0}\right)-\gamma\right]\right\}\right) .
\end{aligned}
$$


Since the second term given by $o\left(\exp \left\{-\lambda^{2} \gamma\right\}\right)$ goes to zero exponentially fast, our main concern is on the first term. Keeping in mind that $\eta_{t}^{\lambda}=\lambda\left(U_{t}^{\lambda}-\phi_{0}(t)\right)$, we have

$$
\begin{aligned}
& \mathbf{E}\left[1_{\left\{\left\|U^{\lambda}-\phi_{0}\right\|<\varepsilon\right\}} \exp \left\{\lambda^{2} F\left(U^{\lambda}\right)\right\}\right] \\
& =\mathbf{E}^{z^{2}}\left[1 _ { \{ \| \eta ^ { \lambda } \| < \varepsilon \lambda \} } \operatorname { e x p } \left\{\lambda^{2} F\left(U^{\lambda}\right)-\int_{0}^{1} z^{\lambda}(t) d U_{t}^{\lambda}\right.\right. \\
& \left.\left.\quad+\lambda^{2} \int_{0}^{1} G_{0}\left(U_{t}^{\lambda} ; z_{0}(t)\right) d t\right\}\right] \\
& =\mathbf{E}\left[1 _ { \{ \| U ^ { 1 } \| < \varepsilon \lambda \} } \operatorname { e x p } \left\{\lambda^{2} F\left(\phi_{0}+\lambda^{-1} U^{1}\right)-\lambda \int_{0}^{1} z_{0}(t) d U_{t}^{1}\right.\right. \\
& \left.\left.\quad-\lambda^{2} \int_{0}^{1}\left(z_{0}(t) \phi_{0}^{\prime}(t)-G_{0}\left(\phi_{0}(t)+\lambda^{-1} U_{t}^{1} ; z_{0}(t)\right)\right) d t\right\}\right] .
\end{aligned}
$$

Now we apply Taylor's expansions to $F$ at the point $\phi_{0}$ up to order $s$ with Peano's remainder

$$
\begin{aligned}
F\left(\phi_{0}+\lambda^{-1} U^{1}\right)= & F\left(\phi_{0}\right)+\lambda^{-1} F^{\prime}\left(\phi_{0}\right)\left(U^{1}\right) \\
& +\cdots+\frac{\lambda^{-s}}{s !} F^{(s)}\left(\phi_{0}\right)\left(U^{1}, \ldots, U^{1}\right)+o\left(\lambda^{-s}\left\|U^{1}\right\|^{s}\right) .
\end{aligned}
$$

Thus (3.2) is equal to

$$
\begin{aligned}
&=\exp \{\left.\lambda^{2}\left[F\left(\phi_{0}\right)-S\left(\phi_{0}\right)\right]\right\} \\
& \times \mathbf{E}\left[1 _ { \{ \| U ^ { 1 } \| < \varepsilon \lambda \} } \operatorname { e x p } \left\{\lambda\left[F^{\prime}\left(\phi_{0}\right)\left(U^{1}\right)-\left(\int_{0}^{1} z_{0}(t) d U_{t}^{1}+\int_{0}^{1} U_{t}^{1} z_{0}(t) d t\right)\right]\right.\right. \\
&+\frac{1}{2} F^{\prime \prime}\left(\phi_{0}\right)\left(U^{1}, U^{1}\right)+\frac{1}{6} \lambda^{-1} F^{\prime \prime \prime}\left(\phi_{0}\right)\left(U^{1}, U^{1}, U^{1}\right) \\
&\left.\left.+\cdots+\frac{\lambda^{-(s-2)}}{s !} F^{(s)}\left(\phi_{0}\right)\left(U^{1}, \ldots, U^{1}\right)+o\left(\lambda^{-(s-2)}\left\|U^{1}\right\|^{s}\right)\right\}\right] .
\end{aligned}
$$

The identity $F^{\prime}\left(\phi_{0}\right)\left(U^{1}\right)-\left(\int_{0}^{1} z_{0}(t) d U_{t}^{1}+\int_{0}^{1} U_{t}^{1} z_{0}(t) d t\right)=0$ (see Appendix (a) for the proof) implies that (3.2) is equal to

$$
\begin{aligned}
=\exp \left\{\lambda^{2}\left[F\left(\phi_{0}\right)-S\left(\phi_{0}\right)\right]\right\} \\
\times \mathbf{E}\left[1 _ { \{ \| U ^ { 1 } \| < \varepsilon \lambda \} } \operatorname { e x p } \left\{\frac{1}{2} F^{\prime \prime}\left(\phi_{0}\right)\left(U^{1}, U^{1}\right)+\frac{1}{6} \lambda^{-1} F^{\prime \prime \prime}\left(\phi_{0}\right)\left(U^{1}, U^{1}, U^{1}\right)\right.\right. \\
\left.\left.\quad+\cdots+\frac{\lambda^{-(s-2)}}{s !} F^{(s)}\left(\phi_{0}\right)\left(U^{1}, \ldots, U^{1}\right)+o\left(\lambda^{-(s-2)}\left\|U^{1}\right\|^{s}\right)\right\}\right] .
\end{aligned}
$$


We now break the set $\left\{\left\|U^{1}\right\|<\varepsilon \lambda\right\}$ into two parts: $\left\{\lambda^{0.1} \leq\left\|U^{1}\right\|<\varepsilon \lambda\right\}$ and $\left\{\left\|U^{1}\right\|<\lambda^{0.1}\right\}$. First we consider the first part $\left\{\lambda^{0.1} \leq\left\|U^{1}\right\|<\varepsilon \lambda\right\}$. For any $\beta>0$, there is a small $\varepsilon>0$ such that

$$
\begin{gathered}
\mathbf{E}\left[1 _ { \{ \lambda ^ { 0 . 1 } \leq \| U ^ { 1 } \| < \varepsilon \lambda \} } \operatorname { e x p } \left\{\frac{1}{2} F^{\prime \prime}\left(\phi_{0}\right)\left(U^{1}, U^{1}\right)+\frac{1}{6} \lambda^{-1} F^{\prime \prime \prime}\left(\phi_{0}\right)\left(U^{1}, U^{1}, U^{1}\right)\right.\right. \\
\left.\left.+\cdots+\frac{\lambda^{-(s-2)}}{s !} F^{(s)}\left(\phi_{0}\right)\left(U^{1}, \ldots, U^{1}\right)+o\left(\lambda^{-(s-2)}\left\|U^{1}\right\|^{s}\right)\right\}\right] \\
\leq \mathbf{E}\left[1_{\left\{\lambda^{0.1} \leq\left\|U^{1}\right\|<\varepsilon \lambda\right\}} \exp \left\{\frac{1}{2} F^{\prime \prime}\left(\phi_{0}\right)\left(U^{1}, U^{1}\right)+\beta\left\|U^{1}\right\|^{2}\right\}\right] \\
\leq\left[\mathbf{P}\left\{\lambda^{0.1} \leq\left\|U^{1}\right\|\right\} \cdot \mathbf{E} \exp \left\{2 \beta\left\|U^{1}\right\|^{2}\right\}\right]^{1 / 2}
\end{gathered}
$$

where in the last inequality the assumption $F^{\prime \prime}\left(\phi_{0}\right)\left(U^{1}, U^{1}\right) \leq 0$ is applied. From Doob's transform, it follows $U_{t}^{1} \sim e^{-t} W_{1 / 2\left(e^{2 t}-1\right)}$ in the sense of distribution. Thus the expectation on the first part $\left\{\lambda^{0.1} \leq\left\|U^{1}\right\|<\varepsilon \lambda\right\}$ tends to zero exponentially fast (see Appendix (b)). So we just need to deal with the expectation on the second part $\left\{\left\|U^{1}\right\|<\lambda^{0.1}\right\}$. Set

$$
\begin{aligned}
a_{\lambda}= & \frac{1}{6} \lambda^{-1} F^{\prime \prime \prime}\left(\phi_{0}\right)\left(U^{1}, U^{1}, U^{1}\right) \\
& +\cdots+\frac{\lambda^{-(s-2)}}{s !} F^{(s)}\left(\phi_{0}\right)\left(U^{1}, \ldots, U^{1}\right)+o\left(\lambda^{-(s-2)}\left\|U^{1}\right\|^{s}\right) .
\end{aligned}
$$

On the set $\left\{\left\|U^{1}\right\|<\lambda^{0.1}\right\}$, noticing that $\lim _{\lambda \rightarrow \infty} a^{\lambda}=0$, we then apply $e^{a_{\lambda}}=$ $1+a_{\lambda}+\cdots+a_{\lambda}^{s-2} /(s-2) !+o\left(a_{\lambda}^{s-2}\right)$ to see that

$$
\begin{aligned}
\mathbf{E}\left[1 _ { \{ \| U ^ { 1 } \| < \lambda ^ { 0 . 1 } \} } \operatorname { e x p } \left\{\frac{1}{2} F^{\prime \prime}\left(\phi_{0}\right)\left(U^{1}, U^{1}\right)+\frac{1}{6} \lambda^{-1} F^{\prime \prime \prime}\left(\phi_{0}\right)\left(U^{1}, U^{1}, U^{1}\right)\right.\right. \\
\left.\left.+\cdots+\frac{\lambda^{-(s-2)}}{s !} F^{(s)}\left(\phi_{0}\right)\left(U^{1}, \ldots, U^{1}\right)+o\left(\lambda^{-(s-2)}\left\|U^{1}\right\|^{s}\right)\right\}\right] \\
=\mathbf{E}\left[1 _ { \{ \| U ^ { 1 } \| < \lambda ^ { 0 . 1 } \} } \operatorname { e x p } \{ \frac { 1 } { 2 } F ^ { \prime \prime } ( \phi _ { 0 } ) ( U ^ { 1 } , U ^ { 1 } ) \} \left(1+\frac{1}{6} \lambda^{-1} F^{\prime \prime \prime}\left(\phi_{0}\right)\left(U^{1}, U^{1}, U^{1}\right)\right.\right. \\
\left.\left.\quad+\cdots+\lambda^{-(s-2)}(\operatorname{sth} .)+o\left(\lambda^{-(s-2)}\left[\left\|U^{1}\right\|^{s}+\left\|U^{1}\right\|^{3(s-2)}\right]\right)\right)\right] \\
=\sum_{0 \leq i \leq(s-2)} K_{i} \lambda^{-i}-\mathbf{E}\left[1_{\left\{\left\|U^{1}\right\| \geq \lambda^{0.1}\right\}} \exp \left\{\frac{1}{2} F^{\prime \prime}\left(\phi_{0}\right)\left(U^{1}, U^{1}\right)\right\}\right. \\
\left.\quad \times\left(1+\frac{1}{6} \lambda^{-1} F^{\prime \prime \prime}\left(\phi_{0}\right)\left(U^{1}, U^{1}, U^{1}\right)+\cdots+\lambda^{-(s-2)} \mathfrak{R}\right)\right] \\
+\mathbf{E}\left[1_{\left\{\left\|U^{1}\right\|<\lambda^{0.1}\right\}} \exp \left\{\frac{1}{2} F^{\prime \prime}\left(\phi_{0}\right)\left(U^{1}, U^{1}\right)\right\} o\left(\lambda^{-(s-2)}\left[\left\|U^{1}\right\|^{s}+\left\|U^{1}\right\|^{3(s-2)}\right]\right)\right] .
\end{aligned}
$$


From a fact in Appendix (c) that $\mathbf{E}\left\|U^{1}\right\|^{j}<\infty$ for any positive integer $j$, it follows

$$
\begin{aligned}
& \mathbf{E}\left[1_{\left\{\left\|U^{1}\right\| \geq \lambda^{0.1}\right\}} \exp \left\{\frac{1}{2} F^{\prime \prime}\left(\phi_{0}\right)\left(U^{1}, U^{1}\right)\right\}\right. \\
& \left.\quad \times\left(1+\frac{1}{6} \lambda^{-1} F^{\prime \prime \prime}\left(\phi_{0}\right)\left(U^{1}, U^{1}, U^{1}\right)+\cdots+\lambda^{-(s-2)} \mathfrak{R}\right)\right]
\end{aligned}
$$

tends to zero exponentially fast, and

$$
\begin{aligned}
& \mathbf{E}\left[1_{\left\{\left\|U^{1}\right\|<\lambda^{0.1}\right\}} \exp \left\{\frac{1}{2} F^{\prime \prime}\left(\phi_{0}\right)\left(U^{1}, U^{1}\right)\right\} o\left(\lambda^{-(s-2)}\left[\left\|U^{1}\right\|^{s}+\left\|U^{1}\right\|^{3(s-2)}\right]\right)\right] \\
& =o\left(\lambda^{-(s-2)}\right) .
\end{aligned}
$$

The proof of Theorem 2.1 is complete.

\subsection{An application to partial differential equations}

Let us recall that the OU process $U^{\lambda}$ defined through $d U_{t}^{\lambda}=-U_{t}^{\lambda} d t+$ $\lambda^{-1} d W_{t}$ has a generating operator $A^{U^{\lambda}}$ given by

$$
A^{U^{\lambda}} f(x)=-x f^{\prime}(x)+\frac{1}{2} \lambda^{-2} f^{\prime \prime}(x)
$$

For a bounded and uniformly continuous function $c(x)$, the following Cauchy problem

$$
\begin{cases}\frac{\partial u^{\lambda}}{\partial t}(t, x)=A^{U^{\lambda}} u^{\lambda}(t, x)+\lambda^{2} c(x) u^{\lambda}(t, x), & t>0, x \in \mathbf{R} \\ u^{\lambda}(0, x)=1, & t=0, x \in \mathbf{R}\end{cases}
$$

can be proved to have a unique solution (in the class of bounded functions) in the form

$$
u^{\lambda}(t, x)=\mathbf{E}_{x} \exp \left\{\lambda^{2} \int_{0}^{t} c\left(U_{s}^{\lambda}\right) d s\right\}
$$

(see Section 1.5 in [4] for more details). Then the solution $u^{\lambda}(t, x)$ of the Cauchy problem has an asymptotic expansion at $(1,0)$ as follows

$$
\begin{aligned}
u^{\lambda}(1,0) & =\mathbf{E} \exp \left\{\lambda^{2} \int_{0}^{1} c\left(U_{s}^{\lambda}\right) d s\right\} \\
& =\exp \left\{\lambda^{2}\left[\int_{0}^{1} c\left(\phi_{0}(s)\right) d s-S\left(\phi_{0}\right)\right]\right\}\left[\sum_{0 \leq i \leq s-2} K_{i} \cdot \lambda^{-i}+o\left(\lambda^{-(s-2)}\right)\right]
\end{aligned}
$$

provided the maximum of $\int_{0}^{1} c(\phi(s)) d s-S(\phi)$ over the space $C_{0}[0,1]$ is reached uniquely at $\phi_{0}$. 


\subsection{Appendix}

In this section we give the details for several facts used in Section 3.2.

(a) The proof of $F^{\prime}\left(\phi_{0}\right)\left(U^{1}\right)-\left(\int_{0}^{1} z_{0}(t) d U_{t}^{1}+\int_{0}^{1} U_{t}^{1} z_{0}(t) d t\right)=0$ is included here. Noticing that $F^{\prime}\left(\phi_{0}\right)$ is a bounded and linear functional on the space $C_{0}[0,1]$, we have

$$
F^{\prime}\left(\phi_{0}\right)(\phi)=\int_{0}^{1} \phi(t) d V(t)
$$

for some right continuous function $V(t)$ of bounded variation on $[0,1]$. Without loss of generality, we impose $V(1)=0$. Since the maximum of $F-S$ is reached uniquely at $\phi_{0}$, for any continuously differentiable function $\phi$, it is true that

$$
F^{\prime}\left(\phi_{0}\right)(\phi)-S^{\prime}\left(\phi_{0}\right)(\phi)=0 .
$$

Thus

$$
\begin{aligned}
0 & =F^{\prime}\left(\phi_{0}\right)(\phi)-S^{\prime}\left(\phi_{0}\right)(\phi)=\int_{0}^{1} \phi(t) d V(t)-\int_{0}^{1}\left(\phi_{0}(t)+\phi_{0}^{\prime}(t)\right)\left(\phi(t)+\phi^{\prime}(t)\right) d t \\
& =-\int_{0}^{1} \phi^{\prime}(t) V(t) d t-\int_{0}^{1}\left[\left(\phi_{0}(t)+\phi_{0}^{\prime}(t)\right)+\int_{t}^{1}\left(\phi_{0}(s)+\phi_{0}^{\prime}(s)\right) d s\right] \phi^{\prime}(t) d t
\end{aligned}
$$

from which it follows

$$
V(t)=-\left(\phi_{0}(t)+\phi_{0}^{\prime}(t)\right)-\int_{t}^{1}\left(\phi_{0}(s)+\phi_{0}^{\prime}(s)\right) d s .
$$

The assumption $V(1)=0$ implies $\phi_{0}(1)+\phi_{0}^{\prime}(1)=0$. Now the proof follows from integration by parts.

(b) Here we prove that $\mathbf{P}\left\{\lambda^{0.1} \leq\left\|U^{1}\right\|\right\}$ tends to zero exponentially fast. From the representation $U_{t}^{1}=e^{-t} W_{(1 / 2)\left(e^{2 t}-1\right)}$, it follows that

$$
\mathbf{P}\left\{\lambda^{0.1} \leq\left\|U^{1}\right\|\right\} \leq \mathbf{P}\left\{\lambda^{0.1} \leq \max _{0 \leq t \leq(1 / 2)\left(e^{2}-1\right)}\left|W_{t}\right|\right\}
$$

which goes to zero exponentially fast as $\lambda \rightarrow \infty$. that

(c) For any constant $\alpha>0$, there is some $\beta(j)>0$ depending only on $j$ such

$$
\mathbf{E}\left\|U^{1}\right\|^{j} \leq \beta(j) \mathbf{E} \exp \left\{\alpha\left\|U^{1}\right\|^{2}\right\} .
$$

Then it follows from the representation $U_{t}^{1}=e^{-t} W_{(1 / 2)\left(e^{2 t}-1\right)}$ that

$$
\mathbf{E}\left\|U^{1}\right\|^{j} \leq \beta(j) \mathbf{E} \exp \left\{\alpha \max _{0 \leq t \leq(1 / 2)\left(e^{2}-1\right)}\left|W_{t}\right|^{2}\right\}<\infty \text { for small } \alpha .
$$

Acknowledgements. The author is grateful to an anonymous referee for the helpful comments and suggestions. 


\section{REFERENCES}

[1] H. Cramér, Sur un nouveau théorème limite de la théorie des probabilités, Act. Sci. et Ind. 736, Hermann, Paris, 1938.

[2] A. Dembo and O. Zeitouni, Large deviations techniques and applications, 2nd ed., Springer, New York, 2010.

[3] R. Ellis AND J. Rosen, Asymptotic analysis of Gaussian integrals, I, Isolated minimum points, Trans. Amer. Math. Soc. 273 (1982), 447-481.

[4] M. Freidlin AND A. Wentzell, Random perturbations of dynamical systems, 2nd ed., Springer, New York, 1998.

[5] V. Piterbarg and V. Fatalov, The Laplace method for probability measures in Banach spaces, Russian Math. Surveys 50 (1995), 1151-1239.

[6] M. Schilder, Some asymptotic formulas for Wiener integrals, Trans. Amer. Math. Soc. 125 (1966), 63-85.

[7] S. R. S. Varadhan, Asymptotic probabilities and differential equations, Comm. Pure Appl. Math. 19 (1969), 261-286.

[8] A. Wentzell, Limit theorems on large deviations for Markov processes, Kluwer, Netherlands, 1990 .

Xiangfeng Yang

School of Mathematics AND CoMputer SCIENCE

Hubei University of Arts and Science

441053 XIANGYANG

P. R. CHINA

CURRENT ADDRESS

Grupo de Fsica Matematica

UNIVERSIDADE DE LISBOA

1649-003 LisBOA

PORTUGAL

E-mail: xyang2@tulane.edu 